\title{
Diet and risk of gastro-oesophageal reflux disease in the Melbourne Collaborative Cohort Study
}

\author{
Sabrina E Wang ${ }^{1,2, *} \odot$, Allison M Hodge ${ }^{2}$, S Ghazaleh Dashti ${ }^{3}$, \\ Suzanne C Dixon-Suen ${ }^{2}$, Hazel Mitchell ${ }^{4}$, Robert JS Thomas ${ }^{5}$, Elizabeth M Williamson ${ }^{6,7}$, \\ Enes Makalic', Alex Boussioutas 5,8, Andrew M Haydon9, Graham G Giles ${ }^{2,10}$, \\ Roger L Milne ${ }^{1,2,10}$, Bradley J Kendall ${ }^{1,12}$ and Dallas R English ${ }^{1,2}$ \\ ${ }^{1}$ Centre for Epidemiology and Biostatistics, Melbourne School of Population and Global Health, The University of \\ Melbourne, Parkville, VIC, Australia: ${ }^{2}$ Cancer Epidemiology Division, Cancer Council Victoria, Melbourne, VIC, \\ Australia: ${ }^{3}$ Clinical Epidemiology and Biostatistics Unit, Murdoch Children's Research Institute, Melbourne, VIC, \\ Australia: ${ }^{4}$ School of Biotechnology and Biomolecular Sciences, University of New South Wales, Kensington, NSW, \\ Australia: ${ }^{5}$ Department of Medicine, Royal Melbourne Hospital, The University of Melbourne, Parkville, VIC, Australia: \\ ${ }^{6}$ Department of Medical Statistics, London School of Hygiene and Tropical Medicine, London, UK: ${ }^{7}$ Health Data \\ Research UK, London, UK: ${ }^{8}$ Victorian Comprehensive Cancer Centre, Parkville, VIC, Australia: ${ }^{9}$ Department of \\ Medical Oncology, Alfred Hospital, Melbourne, VIC, Australia: ${ }^{10}$ Precision Medicine, School of Clinical Sciences at \\ Monash Health, Monash University, Clayłon, VIC, Australia: ${ }^{1}$ Department of Medicine, The University of Queensland, \\ Brisbane, QLD, Australia: ${ }^{12}$ Department of Gastroenterology and Hepatology, Princess Alexandra Hospital, Brisbane, \\ QLD, Australia
}

Submitted 26 October 2020: Final revision received 23 December 2020: Accepted 13 January 2021: First published online 21 January 2021

\begin{abstract}
Objective: To examine associations between diet and risk of developing gastro-oesophageal reflux disease (GERD).

Design: Prospective cohort with a median follow-up of $15 \cdot 8$ years. Baseline diet was measured using a FFQ. GERD was defined as self-reported current or history of daily heartburn or acid regurgitation beginning at least 2 years after baseline. Sex-specific logistic regressions were performed to estimate OR for GERD associated with diet quality scores and intakes of nutrients, food groups and individual foods and beverages. The effect of substituting saturated fat for monounsaturated or polyunsaturated fat on GERD risk was examined.

Setting: Melbourne, Australia.

Participants: A cohort of 20926 participants (62\% women) aged 40-59 years at recruitment between 1990 and 1994 .

Results: For men, total fat intake was associated with increased risk of GERD (OR 1.05 per $5 \mathrm{~g} / \mathrm{d}$; $95 \%$ CI 1.01, 1.09; $P=0.016$ ), whereas total carbohydrate (OR 0.89 per $30 \mathrm{~g} / \mathrm{d} ; 95 \% \mathrm{CI} 0.82,0.98 ; P=0.010)$ and starch intakes (OR 0.84 per $30 \mathrm{~g} / \mathrm{d}$; $95 \%$ CI $0.75,0.94 ; P=0.005)$ were associated with reduced risk. Nutrients were not associated with risk for women. For both sexes, substituting saturated fat for polyunsaturated or monounsaturated fat did not change risk. For both sexes, fish, chicken, cruciferous vegetables and carbonated beverages were associated with increased risk, whereas total fruit and citrus were associated with reduced risk. No association was observed with diet quality scores.

Conclusions: Diet is a possible risk factor for GERD, but food considered as triggers of GERD symptoms might not necessarily contribute to disease development. Potential differential associations for men and women warrant further investigation.
\end{abstract}

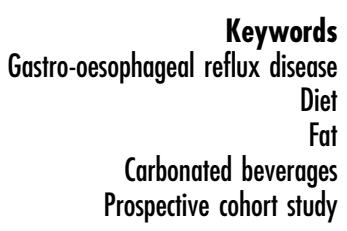

Gastro-oesophageal reflux occurs when gastric contents flow back into the oesophagus. Gastro-oesophageal reflux disease (GERD) is defined when reflux causes troublesome symptoms and/or complications ${ }^{(1)}$. Common symptoms of
GERD include heartburn, a burning sensation in the retrosternal area, and regurgitation, a perception of flow of refluxed gastric contents into the mouth or hypopharynx, together known as the typical reflux syndrome ${ }^{(1)}$. Reflux 
symptoms occurring 2 or more days/week or severe symptoms 1 or more day/week are usually considered troublesome $^{(1)}$. Gastro-oesophageal reflux can also have extraoesophageal manifestations such as dental erosion, laryngitis and asthma ${ }^{(2)}$. If left untreated, GERD is associated with clinically significant impairment in physical and psychosocial functioning ${ }^{(3,4)}$ and predisposes individuals to complications such as Barrett's oesophagus and adenocarcinoma of the oesophagus ${ }^{(5)}$.

A global pooled prevalence study estimated that $14 \%$ of the population experienced at least weekly reflux symptoms ${ }^{(6)}$. Proton pump inhibitors, a class of drug commonly prescribed for managing GERD symptoms, have been among the top five most subsidised prescription drugs in Australia for at least the last decade ${ }^{(7)}$. In the USA, GERD costs approximately \$15-20 billion dollars per year, arising from both direct and indirect costs such as work absenteeism and loss of productivity ${ }^{(8)}$.

Since the 1970s, there has been a sharp increase in incidence of oesophageal adenocarcinoma in Western populations ${ }^{(5,9,10)}$. This warrants further study of its modifiable risk factors, including GERD. In contrast to the extensive evidence on dietary triggers of GERD symptoms, evidence on the role of diet in developing GERD is scant. To our knowledge, only one cohort study, from the Swedish Twin Registry, has examined dietary factors ${ }^{(11)}$. None of the food items examined, including vegetables, fruits, fish, meat, rice, flour-based foods, milk, sandwiches, potatoes and grilled and fried food, was associated with the risk of developing GERD.

We conducted a study of the incidence of GERD within the Melbourne Collaborative Cohort Study. Here, we report on diet in relation to the risk of GERD.

\section{Methods}

\section{The Melbourne Collaborative Cobort Study}

Between 1990 and 1994, 41513 participants (59\% women) aged 40-69 years were recruited ${ }^{(12)}$. Italian- and Greekborn participants were targeted to extend the range of dietary and lifestyle exposures. This analysis was restricted to participants aged 40-59 years at baseline who had no history of cancer (except keratinocyte skin cancers), diabetes mellitus or CVD (including heart attack, stroke and angina) at baseline (Fig. 1). Participants whose total energy intake was in the top or bottom $1 \%$ of total energy intake, or who had missing data for identified confounders, were ineligible, as were Greek-born participants due to their low participation in the wave of follow-up when information on GERD was obtained. A post hoc exclusion was applied to those whose GERD symptoms began before or within 2 years after baseline. This exclusion was based on time of symptom onset reported at follow-up, as no information on GERD symptoms was collected at baseline.
Measurement of baseline diet and characteristics

Demographic and lifestyle information was collected via structured interviews at baseline. Baseline characteristics of interest include age, sex, country of birth (Southern Europe or others), socio-economic position, educational attainment (primary or less, some high school, completed high/technical school or completed degree or diploma), smoking status (never, former or current), alcohol consumption (lifelong abstinence, former or current $<20$, 20-39 or $\geq 40 \mathrm{~g} / \mathrm{d}$ ), leisure-time physical activity (a relative physical activity score based on frequency of vigorous exercise, moderate exercise and walking per week over the last 6 months) and occupational physical activity (moderate-heavy physical exertion at work or no). Socio-economic position was measured using an areabased measure of relative socio-economic disadvantage (the Index of Relative Socioeconomic Disadvantage from the Socio-economic Indexes for Areas), which was calculated from census data and based on a list of indicators for disadvantage such as proportion of household or resident with low annual income, low skilled occupations or unemployment ${ }^{(13)}$. Anthropometric measurements were collected by trained staff, and BMI was calculated.

Dietary data were collected using a self-administered 121-item FFQ, from which total energy intake $(\mathrm{kJ} / \mathrm{d})$ and nutrient intakes $(\mathrm{g} / \mathrm{d})$ were calculated. We selected thirty-two dietary variables for analysis based on dietary components, including beverages, that have been cited as triggers of reflux symptoms ${ }^{(14)}$ or associated with risk of Barrett's oesophagus or oesophageal adenocarcinoma ${ }^{(10)}$. We also examined the Mediterranean Dietary Score (MDS) and the Alternate Heathy Eating Index (AHEI-2010). The MDS was modified from Trichopoulou et al. ${ }^{(15)}$. A score of nine indicates the highest degree of adherence; further details are described elsewhere ${ }^{(16)}$. The AHEI-2010 assesses diet based on foods and nutrients that are predictive of chronic disease risk; a higher score predicts lower risk ${ }^{(17)}$.

Data on past Helicobacterpylori (H.pylori) infection were available for randomly chosen subsets of participants, 583 with daily GERD symptoms and 588 with symptoms <1 d/week. In 2012 and 2019, H. pylori antibodies were measured in baseline plasma that had been stored in liquid $\mathrm{N}_{2}$, using a commercially available immunoblotting kit (Helicoblot 2.1; Genelabs Diagnostics).

\section{Definition and ascertainment of gastro-oesophageal reflux disease}

Information on GERD and use of acid-suppressant medications was collected via computer-assisted telephone interviews between 2007 and 2010. Participants were asked whether they had ever experienced heartburn or regurgitation at least $1 \mathrm{~d} /$ week, and if so, the maximum symptom 


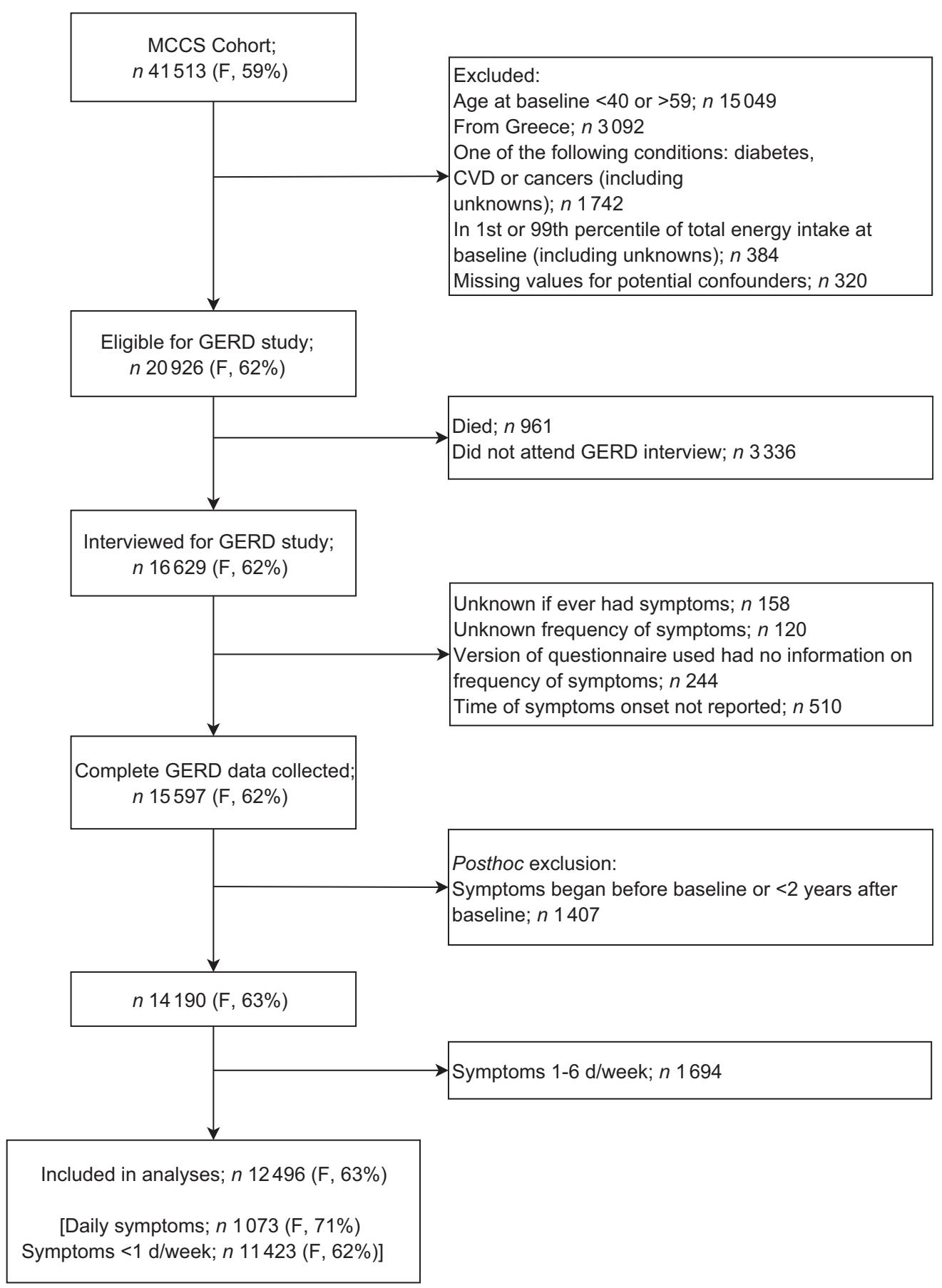

Fig. 1 Participants flow diagram. MCCS, Melbourne Collaborative Cohort Study; GERD, gastro-oesophageal reflux disease; $F$, female

frequency and the time of onset (reported as age at onset, number of years since onset or year of onset). For this analysis, we compared participants with current or past daily heartburn and/or regurgitation (cases) with participants who had symptoms $<1 \mathrm{~d}$ /week or symptoms only during pregnancy (non-cases). Participants with symptoms 1-6 d/week were excluded from analysis to reduce misclassification of the outcome.

\section{Statistical analyses}

Logistic regression was used to estimate the OR and 95\% CI for GERD in relation to the dietary variables. Given that fewer than $10 \%$ of participants had daily GERD symptoms, the OR is a good approximation to the risk ratio ${ }^{(18)}$. In order to address potential selection bias due to missing outcome data (see online Supplemental Table 1), a weighted analysis was performed using the inverse probability weighting 
method with SE estimated using a robust variance estimator $^{(19)}$. Participants were weighted by the inverse of their probability of providing complete GERD data, which was estimated from a logistic prediction model. Participants who completed follow-up and answered all relevant questions on GERD were defined as having provided complete GERD data (Fig. 1). The prediction model was trained using lasso penalised regression ${ }^{(20)}$ with $60 \%$ of the data from eligible participants, and validated using the remaining $40 \%$. Age, sex, country of birth, socio-economic index for area, education, smoking status and alcohol consumption were pre-specified to be included in the prediction model, as their distribution was different for participants with and without missing outcome (see online Supplemental Table 1) and was identified as confounders (see online Supplemental Material 1). The Hosmer-Lemeshow test ${ }^{(19)}$ was performed to assess stability of the weights. The prediction model was trained and validated using the statistical software $\mathrm{R}^{(21)}$. All other analyses were performed using Stata version $14^{(22)}$.

Nutrient intakes were energy-adjusted using the residual method ${ }^{(23)}$. For example, the energy-adjusted fat intake is the residuals from a regression of fat intake on total energy intake. Increments reported are based on approximately 1 SD of intake. In cross-sectional studies, only saturated fat intake has been reported to be associated with GERD $^{(24,25)}$. To investigate the potential effects of intake of different types of fat (saturated, monounsaturated, polyunsaturated) on the risk of GERD, we used Willett's substitution models ${ }^{(23)}$. The first model included energy-adjusted saturated, monounsaturated and polyunsaturated fat and total energy. This assesses whether each type of fat was 'independently' associated with risk of GERD; for intake of one type of fat to vary requires isoenergetic substitution by energy sources other than fat. The second model estimated the potential effect of substituting one type of unsaturated fat with saturated fat while holding the other unsaturated fat intake constant. For instance, to examine the effect of substituting monounsaturated fat with saturated fat, the model included total fat intake, total energy intake, total-fat-adjusted saturated fat and total-fat-adjusted polyunsaturated fat intakes, while monounsaturated fat intake was not included and thus allowed to vary.

Food groups and food items were analysed as approximate quintiles of frequency (times/week) using the lowest quintile as the reference group, and tests for linear trend were performed using the median in each quintile. The MDS and AHEI-2010 were analysed as ordinal variables, using the least adherent category as the reference.

Sex-specific analyses were performed for all dietary exposures. Previous studies have reported that the associations between adiposity and risk of GERD differed between men and women ${ }^{(11,26)}$ and that oestrogen might be involved in the pathogenesis of GERD and oesophagitis in obese women ${ }^{(27)}$. Given that adiposity is a potential intermediate on the causal pathway for the effect of diet on risk of GERD, the association could also be modified by sex. We further examined whether adiposity modifies the association between diet and risk of GERD. Tests for interaction between dietary variables and waist circumference were performed using the likelihood ratio test.

\section{Confounders}

Pre-exposure confounders measured at baseline were identified from the literature and included in a causal diagram (see online Supplemental Material 1). Sex, country of birth, education, socio-economic index for area, occupational and leisure-time physical activity, cigarette smoking and alcohol consumption were included as categorical variables. Age and total energy intake at baseline were included as continuous variables. Dietary confounders specific to certain analyses were also included (Fig. 2 and Table 3). Pearson correlation coefficients were used to examine correlations between dietary exposures. The maximum correlation between variables simultaneously included in regression models was between total fat and total fibre intake $(r=-0.51)$.

\section{Sensitivity analyses}

We performed sensitivity analyses further adjusted for adiposity (measured using waist circumference), asthma or H. pylori infection separately to examine whether results were robust against the three key assumptions shown in the causal diagram (see online Supplemental Material 1). First, the primary analysis assumed adiposity is an intermediate on the causal pathway, instead of a confounder. While it is more likely that diet influences adiposity, it is plausible that in midlife, adiposity affects diet. Second, the primary analysis assumed GERD preceded asthma. There is strong evidence that GERD is an aggravating cofactor that could contribute to asthma development ${ }^{(1,28)}$, but the use of asthma medication has also been associated with increased risk of GERD ${ }^{(29)}$. Third, the primary analysis assumed prior $H$. pylori infection did not affect diet at baseline. However, because prior $H$. pylori infection might influence diet, we adjusted for it in the subset of participants with $H$. pylori assessment.

In addition, we performed analyses aimed at minimising any reverse causation associated with uncertainty in the date of GERD onset and changed diets at baseline in response to non-specific gastric symptoms: (i) by excluding GERD cases with onset within 5 years from baseline; and (ii) by excluding participants who reported following a special diet at baseline.

An analysis with GERD cases redefined as having symptoms $\geq 1 \mathrm{~d}$ /week was performed in order to compare results with the only other cohort study of diet and risk of GERD, which used this definition ${ }^{(11)}$. Last, a complete case (unweighted) analysis was performed. 


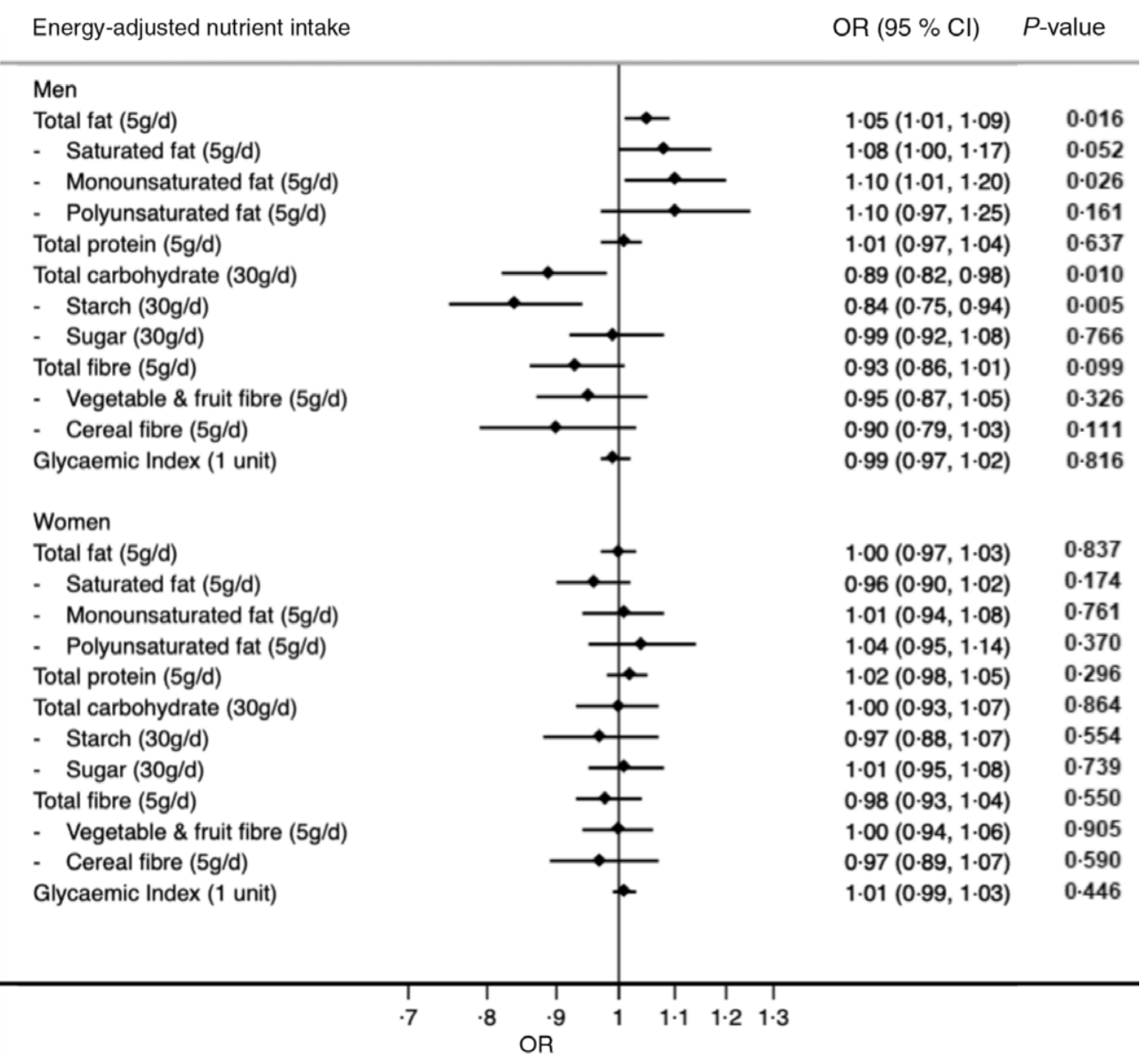

Fig. 2 Estimated OR for nutrient intakes and glycaemic index comparing cases (daily gastro-oesophageal reflux disease (GERD) symptoms) with non-cases (symptoms $<1 \mathrm{~d} /$ week) by sex. Regression models adjusted for age, total energy intake, education, Socioeconomic Indexes for Areas, occupational and leisure physical activity, cigarette smoking, alcohol consumption, country of birth and dietary variables. For the analyses of fat, carbohydrate, protein intake and glycaemic index, the models included total fruit, cruciferous vegetables and leafy vegetables. For the analyses of fibre, the models included total fat

\section{Results}

Of the 20926 initially eligible participants, 16629 (79\%) were interviewed for GERD and 15597 (75\%) provided complete outcome data (Fig. 1). The follow-up time ranged from 12.7 to 19.9 years, with a median of 15.8 years. Response was similar for men and women (see online Supplemental Table 1). Southern Europe-born participants, those with less educational attainment, those who lived in more socio-economically disadvantaged areas and current smokers at baseline, were less likely to provide complete GERD data (see online Supplemental Table 1). Baseline age, total energy intake, waist circumference, BMI and all dietary exposures were similar for those with and without missing GERD data (see online Supplemental Tables 1, 2).

Of the 15597 participants with complete data, 1407 who reported onset of GERD symptoms before or within 2 years from baseline were excluded (Fig. 1). Daily symptoms were reported by 309 men and 764 women, whereas symptoms $<1 \mathrm{~d} /$ week (including symptoms during pregnancy only) were reported by 4331 men and 7091 women (Fig. 1). Daily use of acid-suppressant prescription medication was reported by $60 \%$ of participants who reported daily symptoms, $43 \%$ of participants who reported symptoms 2-6 d/week, $27 \%$ of participants who reported symptoms $1 \mathrm{~d} /$ week and $0.06 \%$ of participants who reported symptoms $<1 \mathrm{~d} /$ week.

Women and participants born in Australia, New Zealand or Northern Europe were more likely to develop daily symptoms than those born in Italy (Table 1). For men, those who had lower educational attainment, were former smokers at baseline, reported light, former or no alcohol consumption, were more physically active for leisure or reported physical exertion at work were more likely to develop daily symptoms. Male cases and non-cases had similar waist circumference and BMI, whereas total energy intake was higher for cases. For women, those who had lower educational attainment, were current smokers at baseline, reported moderate, light, former or no alcohol consumption, were less physically active for leisure or 
Table 1 Baseline characteristics of eligible participants

\begin{tabular}{|c|c|c|c|c|c|c|}
\hline & \multicolumn{3}{|c|}{ Men } & \multicolumn{3}{|c|}{ Women } \\
\hline & GERD $(n$ 309) & $\%(3.9)$ & Total $(n$ 8010) & GERD $(n 764)$ & $\%(5 \cdot 9)$ & $\begin{array}{c}\text { Total } \\
(n 12916) \\
\end{array}$ \\
\hline \multicolumn{7}{|l|}{ Country of birth } \\
\hline Australia/New Zealand/Northern Europe & 278 & 4.0 & 6911 & 666 & $6 \cdot 0$ & 11160 \\
\hline Southern Europe & 31 & $2 \cdot 8$ & 1099 & 98 & $5 \cdot 6$ & 1756 \\
\hline \multicolumn{7}{|l|}{ SEIFA quintiles } \\
\hline 1 (most disadvantaged) & 51 & 4.5 & 1130 & 128 & 6.5 & 1957 \\
\hline 2 & 43 & 3.0 & 1456 & 145 & 5.9 & 2446 \\
\hline 3 & 61 & 4.6 & 1333 & 127 & $6 \cdot 1$ & 2087 \\
\hline 4 & 73 & 4.6 & 1602 & 138 & $5 \cdot 6$ & 2477 \\
\hline 5 & 81 & $3 \cdot 3$ & 2489 & 226 & $5 \cdot 7$ & 3949 \\
\hline \multicolumn{7}{|l|}{ Level of education } \\
\hline Primary school or less & 13 & $2 \cdot 2$ & 603 & 78 & $5 \cdot 7$ & 1371 \\
\hline Some high/technical school & 106 & 4.4 & 2433 & 365 & $6 \cdot 5$ & 5597 \\
\hline Completed high/technical school & 86 & $4 \cdot 1$ & 2078 & 150 & $6 \cdot 0$ & 2487 \\
\hline Completed tertiary education & 104 & $3 \cdot 6$ & 2896 & 171 & 4.9 & 3461 \\
\hline \multicolumn{7}{|l|}{ Cigarette smoking } \\
\hline Never & 144 & 3.7 & 3893 & 492 & $5 \cdot 8$ & 8443 \\
\hline Former & 125 & $4 \cdot 2$ & 2993 & 191 & $6 \cdot 0$ & 3159 \\
\hline Current & 40 & 3.6 & 1124 & 81 & $6 \cdot 2$ & 1314 \\
\hline \multicolumn{7}{|l|}{ Alcohol consumption } \\
\hline Lifetime abstainers & 38 & 4.4 & 870 & 242 & $6 \cdot 3$ & 3856 \\
\hline Ex-drinkers & 37 & 4.0 & 919 & 69 & $6 \cdot 0$ & 1152 \\
\hline$<20 \mathrm{~g} / \mathrm{d}$ & 152 & $4 \cdot 3$ & 3535 & 362 & $5 \cdot 7$ & 6400 \\
\hline $20-39 \mathrm{~g} / \mathrm{d}$ & 48 & $3 \cdot 1$ & 1551 & 72 & $6 \cdot 3$ & 1146 \\
\hline$\geq 40 \mathrm{~g} / \mathrm{d}$ & 34 & 3.0 & 1135 & 19 & $5 \cdot 2$ & 362 \\
\hline \multicolumn{7}{|l|}{ Leisure-time physical activity } \\
\hline 1 (least active) & 63 & 3.7 & 1716 & 178 & $6 \cdot 6$ & 2693 \\
\hline 2 & 57 & 3.8 & 1498 & 166 & $6 \cdot 2$ & 2699 \\
\hline 3 & 83 & 3.6 & 2317 & 240 & $5 \cdot 4$ & 4458 \\
\hline 4 & 106 & $4 \cdot 3$ & 2479 & 180 & 5.9 & 3066 \\
\hline \multicolumn{7}{|l|}{ Physical exertion at work } \\
\hline No & 247 & 3.7 & 6589 & 683 & $5 \cdot 8$ & 11699 \\
\hline \multirow[t]{2}{*}{ Yes } & 62 & 4.4 & 1421 & 81 & $6 \cdot 7$ & 1217 \\
\hline & \multicolumn{2}{|c|}{ GERD $(n$ 309) } & $\begin{array}{c}\text { Non-cases } \\
(n \text { 4332) }\end{array}$ & \multicolumn{2}{|c|}{ GERD $(n$ 764) } & $\begin{array}{c}\text { Non-cases } \\
(n \text { 7091) }\end{array}$ \\
\hline Median age (years) & \multicolumn{2}{|l|}{$48 \cdot 2$} & $48 \cdot 8$ & \multicolumn{2}{|l|}{$48 \cdot 2$} & $49 \cdot 4$ \\
\hline 25th, 75th percentile & \multicolumn{2}{|c|}{$43 \cdot 2,53 \cdot 2$} & $43 \cdot 9,54 \cdot 1$ & \multicolumn{2}{|c|}{$43 \cdot 7,54 \cdot 0$} & $44 \cdot 7,54 \cdot 6$ \\
\hline Median total energy intake $(\mathrm{kJ} / \mathrm{d})$ & \multicolumn{2}{|c|}{$9716 \cdot 8$} & $9319 \cdot 9$ & \multicolumn{2}{|c|}{$7967 \cdot 1$} & $7849 \cdot 9$ \\
\hline 25th, 75th percentile & \multirow{2}{*}{\multicolumn{2}{|c|}{$\begin{array}{c}7581 \cdot 7,12031 \cdot 8 \\
91.5\end{array}$}} & $7538 \cdot 0,11460 \cdot 5$ & \multicolumn{2}{|c|}{$6330 \cdot 0,10099 \cdot 0$} & $6324 \cdot 3,9715 \cdot 2$ \\
\hline Median waist circumference $(\mathrm{cm})$ & & & $90 \cdot 7$ & \multicolumn{2}{|c|}{77} & 74.7 \\
\hline 25th, 75th percentile & \multicolumn{2}{|c|}{$85 \cdot 0,97 \cdot 2$} & $85 \cdot 0,96 \cdot 7$ & \multicolumn{2}{|c|}{$70 \cdot 5,84 \cdot 0$} & $69 \cdot 0,82 \cdot 3$ \\
\hline Median BMI (kg/m²) & \multicolumn{2}{|c|}{$\begin{array}{c}26 \cdot 3 \\
24.428 .5\end{array}$} & $26 \cdot 2$ & $25 \cdot 8$ & & 24.5 \\
\hline 25th, 75th percentile & $24 \cdot 4,28$ & & $24 \cdot 2,28 \cdot 4$ & $23 \cdot 3,2 \varepsilon$ & & $22 \cdot 4,27 \cdot 7$ \\
\hline
\end{tabular}

GERD, gastro-oesophageal reflux disease; SEIFA, socio-economic index for area.

reported physical exertion at work were more likely to develop daily symptoms. Female cases had higher total energy intake, waist circumference and BMI compared with non-cases. The median age at baseline was 48 years for both male and female cases. The median age at symptom onset was 58 years for men and 59 years for women.

For men, cases had higher intakes of fat, meat and dairy, and lower intakes of carbohydrate, fibre and citrus compared with non-cases (Table 2). For women, cases had higher consumption of tea and lower consumption of citrus and coffee compared with non-cases
(Table 2). For both men and women, degree of adherence to the MDS and AHEI-2010 was similar for cases and non-cases.

\section{Nutrient intakes}

For men, total fat intake was associated with increased risk of GERD (OR 1.05 per $5 \mathrm{~g} / \mathrm{d}$; $95 \%$ CI 1.01, 1.09; $P=0.016$ ) and an association was observed for all three types of fat (Fig. 2). Increasing intakes of total carbohydrate (OR $0 \cdot 89$ per $30 \mathrm{~g} / \mathrm{d} ; 95 \%$ CI $0.82,0.98 ; P=0.010)$ and starch (OR 0.84 per $30 \mathrm{~g} / \mathrm{d} ; 95 \%$ CI 0.75, 0.94; $P=0.005)$, but not sugar 


\begin{tabular}{|c|c|c|c|c|c|c|c|c|}
\hline & \multicolumn{4}{|c|}{ Men } & \multicolumn{4}{|c|}{ Women } \\
\hline & \multicolumn{2}{|c|}{ GERD ( $n$ 309) } & \multicolumn{2}{|c|}{ Non-cases ( $n$ 4332) } & \multicolumn{2}{|c|}{ GERD $(n 764)$} & \multicolumn{2}{|c|}{ Non-cases ( $n$ 7091) } \\
\hline & Median & $\begin{array}{l}\text { 25th, 75th } \\
\text { percentile }\end{array}$ & Median & $\begin{array}{l}\text { 25th, 75th } \\
\text { percentile }\end{array}$ & Median & $\begin{array}{l}\text { 25th, 75th } \\
\text { percentile }\end{array}$ & Median & $\begin{array}{l}\text { 25th, 75th } \\
\text { percentile }\end{array}$ \\
\hline \multicolumn{9}{|l|}{ Median energy-adjusted nutrient intake $(\mathrm{g} / \mathrm{d}$ ) } \\
\hline Total fat & $86 \cdot 8$ & $78 \cdot 2,94 \cdot 1$ & 83.4 & $75 \cdot 0,91 \cdot 7$ & $81 \cdot 8$ & $73 \cdot 2,89 \cdot 1$ & $81 \cdot 2$ & $73 \cdot 0,88 \cdot 6$ \\
\hline Saturated fat & 34.4 & $31 \cdot 2,38 \cdot 4$ & 33.4 & $29 \cdot 4,37.4$ & 32.9 & $29 \cdot 1,36 \cdot 4$ & 33.0 & $29.5,36.5$ \\
\hline Monounsaturated fat & 31.0 & $27.4,33.9$ & 29.7 & $26 \cdot 3,33 \cdot 1$ & 28.6 & $25 \cdot 3,31 \cdot 7$ & $28 \cdot 3$ & $25 \cdot 2,31 \cdot 2$ \\
\hline Polyunsaturated fat & $12 \cdot 6$ & $10 \cdot 6,16 \cdot 0$ & $12 \cdot 3$ & $10 \cdot 3,15 \cdot 3$ & $12 \cdot 2$ & $10 \cdot 1,15 \cdot 4$ & $12 \cdot 1$ & $10 \cdot 0,15 \cdot 0$ \\
\hline Total protein & $98 \cdot 1$ & $89 \cdot 3,107 \cdot 3$ & $97 \cdot 8$ & $89 \cdot 1,106 \cdot 8$ & 95.9 & $89.0,103.5$ & 95.7 & $88 \cdot 8,103 \cdot 3$ \\
\hline Total carbohydrate & 239.9 & $219 \cdot 8,260.9$ & 248.0 & $225 \cdot 8,270 \cdot 1$ & 247.1 & $230 \cdot 1,267.5$ & 249.7 & $231.5,268.7$ \\
\hline Starch & $122 \cdot 0$ & $105 \cdot 7,139 \cdot 7$ & $125 \cdot 3$ & $110 \cdot 0,142 \cdot 6$ & $117 \cdot 7$ & $103.9,133.5$ & 117.4 & $104.9,131.9$ \\
\hline Sugar & 112.2 & $92 \cdot 2,137 \cdot 1$ & $118 \cdot 3$ & $95 \cdot 5,141.0$ & $126 \cdot 2$ & $107 \cdot 1,146 \cdot 4$ & $127 \cdot 2$ & $107.4,148.5$ \\
\hline Total fibre & 27.3 & $23 \cdot 4,32 \cdot 1$ & $29 \cdot 2$ & $24.4,34.7$ & 30.2 & $25.4,34.7$ & 30.6 & $26 \cdot 4,35 \cdot 3$ \\
\hline Vegetable/Fruit fibre & 11.2 & $7 \cdot 6,15 \cdot 1$ & $12 \cdot 0$ & $8 \cdot 5,16 \cdot 7$ & $14 \cdot 0$ & $10 \cdot 0,18 \cdot 4$ & $14 \cdot 2$ & $10 \cdot 7,18 \cdot 5$ \\
\hline Cereal fibre & 11.4 & $8 \cdot 7,14 \cdot 2$ & $12 \cdot 0$ & $9 \cdot 3,15 \cdot 0$ & $11 \cdot 1$ & $8 \cdot 8,13.8$ & 11.4 & $9 \cdot 1,13.9$ \\
\hline Median glycaemic index & $49 \cdot 7$ & $46 \cdot 9,52 \cdot 0$ & $49 \cdot 0$ & $46 \cdot 7,52 \cdot 0$ & $49 \cdot 0$ & $46 \cdot 4,52 \cdot 0$ & 48.5 & $46 \cdot 0,51 \cdot 2$ \\
\hline \multicolumn{9}{|l|}{ Median food group (times/week) } \\
\hline Total meat & $12 \cdot 0$ & $9 \cdot 0,16 \cdot 0$ & 11.5 & $8.5,15.5$ & 11.0 & $8.0,14.0$ & $10 \cdot 5$ & $8 \cdot 0,13 \cdot 5$ \\
\hline Red meat & 8.5 & $5.5,12 \cdot 0$ & 8.0 & $5.5,11.0$ & 6.5 & $4.5,9.0$ & 6.5 & $4.5,9 \cdot 0$ \\
\hline Fish & 1.5 & $1 \cdot 0,2 \cdot 0$ & 1.5 & $1 \cdot 0,2 \cdot 0$ & 1.5 & $1.0,2.0$ & 1.5 & $1 \cdot 0,2 \cdot 0$ \\
\hline Chicken & 1.5 & $1.0,3.0$ & 1.5 & $1.0,3.0$ & 2.0 & $1.0,3.5$ & $2 \cdot 0$ & $1.0,3 \cdot 0$ \\
\hline Processed meat & 2.5 & $1.5,4.5$ & 2.5 & $1.5,4.5$ & 2.0 & $1.5,3.5$ & 2.0 & $1.5,3.5$ \\
\hline Total vegetables & $28 \cdot 0$ & $21 \cdot 0,42 \cdot 0$ & 28.0 & $21 \cdot 0,42 \cdot 0$ & $35 \cdot 0$ & $28 \cdot 0,49 \cdot 0$ & $35 \cdot 0$ & $28 \cdot 0,49 \cdot 0$ \\
\hline Leafy vegetables & 4.0 & $2.5,7.5$ & 4.0 & $2 \cdot 0,7 \cdot 0$ & 5.5 & $3 \cdot 0,9 \cdot 2$ & $5 \cdot 0$ & $2.5,8.0$ \\
\hline Cruciferous veqetables & 3.5 & $1.0,4.0$ & 3.5 & $1.5,6.0$ & 4.0 & $2 \cdot 0,6 \cdot 5$ & 4.0 & $2 \cdot 0,6.5$ \\
\hline Total fruit & 21.0 & $14 \cdot 0,28 \cdot 0$ & 21.0 & $14 \cdot 0,35 \cdot 0$ & 28.0 & $14 \cdot 0,35 \cdot 0$ & 28.0 & $14.0,35 \cdot 0$ \\
\hline Total dairy & $36 \cdot 0$ & $19 \cdot 0,49 \cdot 0$ & 33.0 & $18.5,47.5$ & 36.5 & $20 \cdot 5,50 \cdot 5$ & $37 \cdot 0$ & $22.5,50.5$ \\
\hline High-fat/added-sugar discretionary food ${ }^{*}$ & 7.5 & $3.5,14.6$ & $7 \cdot 1$ & $3 \cdot 0,12 \cdot 5$ & 6.4 & $2 \cdot 5,11 \cdot 3$ & $6 \cdot 2$ & $2 \cdot 5,11 \cdot 0$ \\
\hline \multicolumn{9}{|l|}{ Median food item (times/week) } \\
\hline Citrus & 4.1 & $1 \cdot 1,8 \cdot 0$ & $6 \cdot 0$ & $2 \cdot 0,9 \cdot 0$ & 4.5 & $1.5,8.5$ & $6 \cdot 1$ & $2 \cdot 0,10 \cdot 1$ \\
\hline Tomato & 3.0 & $0.5,3.0$ & 3.0 & $0.5,3.0$ & 3.0 & $1.0,5 \cdot 5$ & 3.0 & $1 \cdot 0,5 \cdot 5$ \\
\hline Chocolate & 1.0 & $0.1,3.0$ & 0.5 & $0.1,3.0$ & 0.5 & $0.1,3.0$ & 0.5 & $0.1,1.0$ \\
\hline Carbonated beverages & $1 \cdot 1$ & $0.6,5.5$ & 1.0 & $0.1,3 \cdot 1$ & 0.6 & $0.1,3.1$ & 0.6 & $0.1,2 \cdot 0$ \\
\hline Tea & $7 \cdot 0$ & $1.0,17.5$ & $7 \cdot 0$ & $0.5,17.5$ & 17.5 & $0.5,17.5$ & $7 \cdot 0$ & $1.0,17.5$ \\
\hline Coffee & 17.5 & 3.0 .17 .5 & 17.5 & $3.0,17.5$ & 7.0 & $1.0,17.5$ & 17.5 & $3.0,17.5$ \\
\hline \multicolumn{9}{|l|}{ Median dietary score } \\
\hline Mediterranean diet score & 4 & 3,6 & 4 & 3,6 & 4 & 3,6 & 4 & 3,6 \\
\hline Alternate healthy eating index-2010 & 61 & 54.68 & 61 & 54.69 & 66 & 59,73 & 67 & 60.74 \\
\hline
\end{tabular}

*High-fat added-sugars discretionary food included ice cream, sweet biscuits, cakes or sweet pastries, puddings and chocolate confectionary. 
Diet and gastro-oesophageal reflux disease

(a) Type of fat $(5 g / d)$

OR $(95 \% \mathrm{Cl}) \quad P$-value

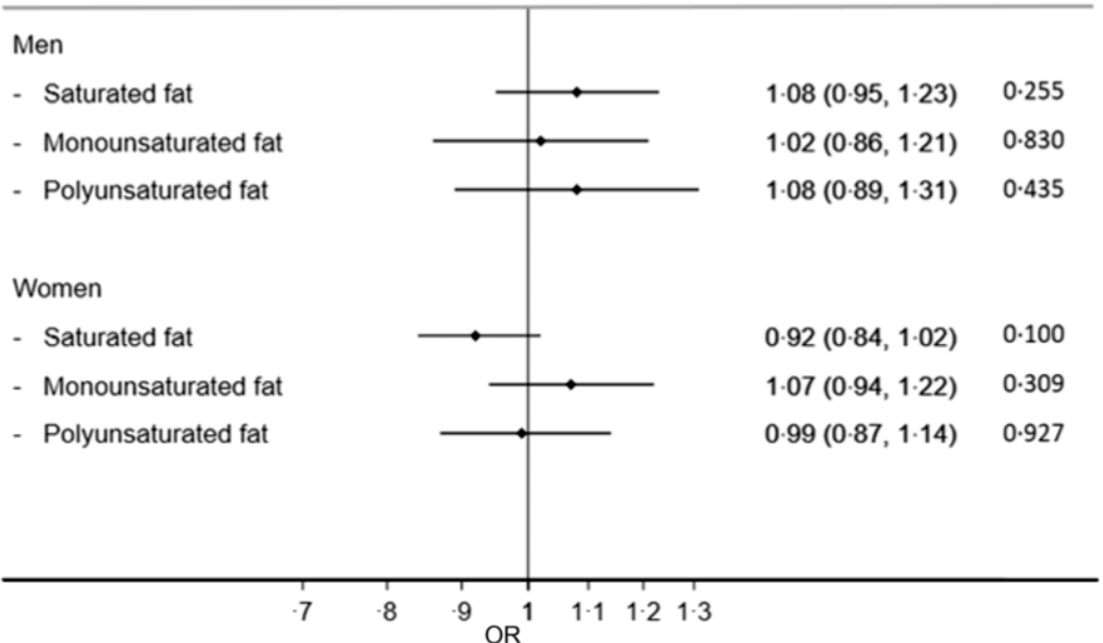

(b) Fat

substitution $(5 g / d)$

OR $(95 \% \mathrm{Cl}) \quad P$-value

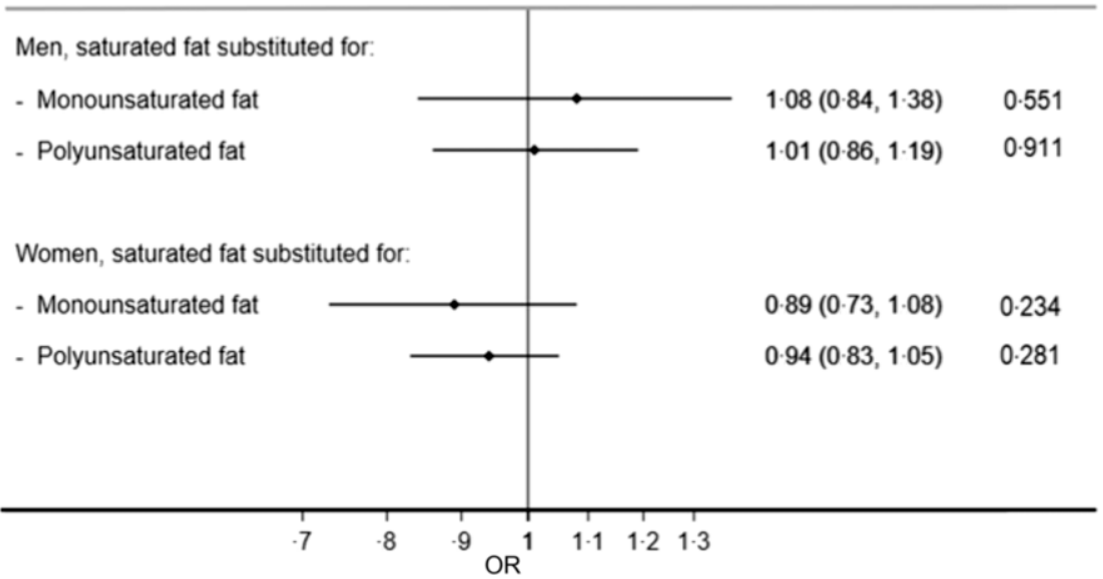

Fig. 3 Estimated OR comparing cases (daily gastro-oesophageal reflux disease (GERD) symptoms) with non-cases (symptoms $<1 \mathrm{~d} /$ week) by sex when (a) each type of fat was substituted for other sources of energy and when (b) saturated fat was substituted for monounsaturated or polyunsaturated fat. Estimates are adjusted for non-dietary and dietary confounders as per Figure 2

or glycaemic index, was associated with reduced risk of GERD. It was impossible to separate the associations for fat and carbohydrate intake, as these nutrients were strongly inversely correlated $(r=-0 \cdot 90)$. Fibre intake was also weakly inversely associated with risk (OR 0.93 per $5 \mathrm{~g} / \mathrm{d} ; 95 \%$ CI $0.86,1.01 ; P=0.099)$. For women, there was little evidence of association between intake of any nutrient and risk of GERD (Fig. 2).

\section{Fat substitutions}

For both men and women, there was no evidence that substituting other sources of energy with individual types of fat (saturated fat, monounsaturated fat or polyunsaturated fat) was associated with risk of GERD (Fig. 3a). This is reflected in the fat substitution model, where substituting monounsaturated fat or polyunsaturated fat with saturated fat did not result in change in GERD risk (Fig. 3b).

\section{Food groups, food items and dietary patterns}

The results from sex-specific analysis for foods and dietary patterns did not differ substantially from those combining the sexes (see online Supplemental Table 3), and thus combined results are presented here. More frequent consumption of fish (OR 1.09 per time/week; $95 \%$ CI 1.03, 1.15 ; trend $P=0.002$ ) and chicken (OR 1.08 per time/week; $95 \%$ CI 1.03, 1.14; trend $P=0.002$ ) was associated with increased risk of GERD, whereas total meat, red meat and processed meat intakes were not (Table 3 ). Total vegetable 
Table 3 OR for food groups, food items and dietary patterns, comparing cases (daily GERD symptoms) with non-cases (symptoms $<1 \mathrm{~d} /$ week)

\begin{tabular}{|c|c|c|c|c|c|c|c|c|c|c|c|c|}
\hline & \multicolumn{8}{|c|}{ Quintiles of consumption frequency ${ }^{*}$} & & & \multirow{3}{*}{$\begin{array}{c}\text { Test for } \\
\text { trend, } \\
P \text {-value }\end{array}$} & \multirow{3}{*}{$\begin{array}{c}\text { Test for } \\
\text { linearity, } \\
P \text {-value }\end{array}$} \\
\hline & \multicolumn{2}{|c|}{ Quintile 2} & \multicolumn{2}{|c|}{ Quintile 3} & \multicolumn{2}{|c|}{ Quintile 4} & \multicolumn{2}{|c|}{ Quintile 5} & \multicolumn{2}{|c|}{$\begin{array}{l}\text { Increase of } \\
1 \text { time/week }\end{array}$} & & \\
\hline & OR & $95 \% \mathrm{Cl}$ & OR & $95 \% \mathrm{Cl}$ & OR & $95 \% \mathrm{Cl}$ & OR & $95 \% \mathrm{Cl}$ & OR & $95 \% \mathrm{Cl}$ & & \\
\hline \multicolumn{13}{|l|}{ Food group } \\
\hline Total meat & 0.99 & $0.81,1.21$ & 1.09 & $0.90,1.33$ & 1.08 & $0.87,1.34$ & $1 \cdot 13$ & $0.90,1.41$ & 1.01 & $0.99,1.03$ & 0.202 & 0.820 \\
\hline Red meat & 1.05 & $0.86,1.28$ & 0.93 & $0.76,1.13$ & 0.88 & $0.72,1.08$ & 0.98 & $0.79,1.21$ & 0.99 & $0.97,1.01$ & 0.537 & 0.434 \\
\hline Fish & 0.98 & $0.80,1.19$ & 1.00 & $0.81,1.22$ & $1 \cdot 17$ & $0.96,1.43$ & 1.30 & $1.05,1.61$ & 1.09 & $1.03,1.15$ & 0.002 & 1.000 \\
\hline Chicken & 1.07 & $0.89,1.29$ & 1.06 & $0.86,1.30$ & $1 \cdot 13$ & $0.93,1.38$ & 1.34 & $1.11,1.63$ & 1.08 & $1.03,1.14$ & 0.002 & 0.852 \\
\hline Processed meat & 1.01 & $0.83,1.22$ & 1.09 & $0.86,1.37$ & 0.94 & $0.79,1.12$ & 0.99 & $0.81,1.20$ & 0.99 & $0.96,1.03$ & 0.749 & 0.584 \\
\hline Total vegetables & 0.95 & $0.78,1 \cdot 17$ & 0.97 & $0.81,1 \cdot 16$ & 1.06 & $0.83,1.34$ & 1.08 & $0.88,1.32$ & 1.00 & $1.00,1.01$ & 0.290 & 0.899 \\
\hline Leafy vegetables & 0.98 & $0.82,1.17$ & 0.89 & $0.71,1.12$ & 0.96 & $0.78,1.17$ & 0.95 & $0.78,1.17$ & 0.99 & $0.97,1.02$ & 0.661 & 0.904 \\
\hline Cruciferous vegetables & 0.99 & $0.80,1.22$ & 1.23 & $0.99,1.51$ & 1.23 & $1.00,1.50$ & 1.48 & $1.20,1.82$ & 1.04 & $1.02,1.06$ & $<0.001$ & 0.506 \\
\hline Total fruit & 0.79 & $0.65,0.95$ & 0.80 & $0.65,0.97$ & 0.78 & $0.64,0.95$ & 0.73 & $0.58,0.92$ & 0.99 & $0.99,1.00$ & 0.012 & 0.187 \\
\hline Total dairy & 0.81 & $0.66,1.00$ & 0.87 & $0.70,1.06$ & 0.91 & $0.74,1.12$ & 0.80 & $0.64,1.00$ & 1.00 & $0.99,1.00$ & 0.173 & 0.236 \\
\hline $\begin{array}{l}\text { High-fat/added-sugar discretionary } \\
\text { food }\end{array}$ & 0.98 & $0.80,1.20$ & 0.93 & $0.75,1.15$ & 0.94 & $0.75,1.16$ & 1.07 & $0.86,1.34$ & 1.00 & $0.99,1.01$ & 0.386 & 0.611 \\
\hline \multicolumn{13}{|l|}{ Food item } \\
\hline Citrus & 0.83 & $0.68,1.00$ & 0.87 & $0.72,1.05$ & 0.59 & $0.48,0.74$ & 0.66 & $0.53,0.81$ & 0.97 & $0.95,0.98$ & $<0.001$ & 0.018 \\
\hline Tomato & 0.88 & $0.72,1.06$ & 0.82 & $0.69,0.97$ & 0.90 & $0.71,1.14$ & 0.89 & $0.71,1 \cdot 12$ & 0.99 & $0.96,1.02$ & 0.430 & 0.209 \\
\hline Chocolate & 0.92 & $0.76,1.10$ & 1.00 & $0.83,1.20$ & 0.97 & $0.79,1.20$ & $1 \cdot 10$ & $0.87,1.41$ & 1.02 & $0.98,1.05$ & 0.302 & 0.752 \\
\hline Carbonated beverages & 1.00 & $0.82,1.23$ & $1 \cdot 18$ & $0.96,1.45$ & 1.30 & $1.06,1.58$ & 1.48 & $1 \cdot 24,1 \cdot 77$ & 1.06 & $1.03,1.08$ & $<0.001$ & 0.741 \\
\hline Tea & 0.78 & $0.63,0.97$ & 0.87 & $0.70,1.08$ & 0.90 & $0.74,1.09$ & 1.01 & $0.82,1.23$ & 1.00 & $1.00,1.01$ & 0.241 & 0.094 \\
\hline Coffee & 0.95 & $0.76,1.19$ & 0.79 & $0.63,1.00$ & 0.87 & $0.71,1.06$ & 0.89 & $0.72,1.12$ & 1.00 & $0.99,1.00$ & 0.544 & 0.272 \\
\hline MDS & \multicolumn{2}{|c|}{ Score 4-6 } & \multicolumn{2}{|c|}{ Score 7-9 } & & & \multicolumn{6}{|c|}{$\begin{array}{l}\text { Increase of one } \\
\text { score }\end{array}$} \\
\hline Score 0-3 (ref) & 0.91 & $0.78,1.05$ & 0.92 & $0.73,1.17$ & & & & & 0.99 & $0.94,1.03$ & 0.507 & 0.129 \\
\hline AHEI-2010 & \multicolumn{2}{|c|}{ Quintile 2} & \multicolumn{2}{|c|}{ Quintile 3} & \multicolumn{2}{|c|}{ Quintile 4} & \multicolumn{2}{|c|}{ Quintile 5} & \multicolumn{2}{|c|}{$\begin{array}{l}\text { Increase of one } \\
\text { score }\end{array}$} & & \\
\hline Quintile 1 (ref) & 1.01 & $0.82,1.24$ & 1.05 & $0.85,1.29$ & 1.07 & $0.87,1.32$ & 0.90 & $0.71,1.12$ & 1.00 & $0.99,1.01$ & 0.624 & 0.296 \\
\hline
\end{tabular}

MDS, Mediterranean Diet Score; AHEI-2010, Alternate Healthy Eating Index-2010.

*Estimated OR comparing each quintile of consumption frequency with the lowest quintile. Regression models adjusted for age total energy intake, education, Socio-economic Indexes for Areas, occupational and leisure physical activity cigarette smoking, alohol consumption, country of birth and dian

fruit, cruciferous vegetables and leafy vegetables. For the analyses of vegetable and fruit intake, the models included total fat. 
consumption and leafy vegetable consumption were not associated with GERD, whereas consumption of cruciferous vegetables was associated with increased risk (OR 1.04 per time/week; $95 \%$ CI 1.02, 1.06; trend $P<0.001)$. For total fruit intake, reduced risk was observed across all quintiles compared with the lowest quintile of consumption frequency (Q5 v. Q1: OR 0.73; $95 \%$ CI 0.58, 0.92; trend $P=0.012$ ). Citrus fruit consumption was associated with reduced risk of GERD (OR 0.97 per time/week; $95 \%$ CI 0.95, 0.98; trend $P<0.001)$. Carbonated beverages were associated with increased risk (OR 1.06 per time/week, $95 \%$ CI 1.03, 1.08; trend $P<0.001)$. There was no evidence that consumption of dairy, tea, coffee, chocolate, high-fat added-sugar discretionary food or tomato was associated with the risk of GERD. Neither the MDS nor the AHEI-2010 was associated with risk.

\section{Dietary factors and adiposity}

There was no evidence that the association between dietary factors and risk of GERD was modified by waist circumference (see online Supplemental Table 4).

\section{Sensitivity analyses}

Further adjustment for waist circumference or asthma had no influence on the results (see online Supplemental Tables 5 and 6). In the subset of participants with $H$. pylori measurements, adjustment for $H$. pylori status also had no influence (see online Supplemental Table 7). Excluding participants who reported GERD onset within 5 years from baseline did not change the study results (see online Supplemental Table 8). When analysis was restricted to people who reported not following a special diet at baseline, the association for fish, chicken and total fruit was attenuated (see online Supplemental Table 9). Results from the complete case (unweighted) analysis (see online Supplemental Table 10) did not differ from the analysis using the inverse probability weighting method, suggesting that missing outcome data did not have a material effect on the results. When the case definition was redefined as symptoms $\geq 1 \mathrm{~d} /$ week, all observed associations, except for chicken, weakened; the greatest attenuation was observed for fat and carbohydrate intake in men, although starch and cereal fibre remained inversely associated with GERD (see online Supplemental Table 11).

\section{Discussion}

For men, fat intake was associated with increased GERD risk, while carbohydrate intake, specifically intake of starch, was associated with reduced risk. The strong negative correlation between fat and carbohydrate intakes did not allow attributing the association to either macronutrient. No nutrient was associated with risk for women. For both sexes, substituting monounsaturated or polyunsaturated fat with saturated fat did not change GERD risk.
For both sexes combined, higher frequencies of consuming fish, chicken, cruciferous vegetables and carbonated beverages were associated with increased risk of GERD, while total fruit and citrus were inversely associated with risk. There was no evidence of association with MDS or AHEI-2010. There was no evidence of interaction between dietary factors and adiposity.

Comprehensive data on demographic and lifestyle factors allowed adjustment for pre-exposure confounders, although residual confounding due to unmeasured or poorly measured variables is possible. Complete data on GERD were missing for $25 \%$ of eligible participants, but the results from inverse probability weighted and unweighted analyses were similar. The inverse probability weighting method assumes data were missing at random (i.e. the probability that data are missing depends only on observed data). We cannot rule out that there might be unobserved variables that are predictive of missingness. Although the assumption is unverifiable, effort was made to include key variables in the prediction model. While our study was not strictly prospective because information on GERD was obtained after baseline, exclusion of participants whose symptoms began before or within 5 years after measurement of diet suggested that reverse causality due to pre-baseline GERD, a feature of cross-sectional studies, is unlikely to explain the associations.

The large sample size permitted estimation of sexspecific associations and allowed us to contrast the maximum (daily) and minimum ( $<1 \mathrm{~d} /$ week) symptom frequency to improve the specificity of our outcome definition. Cross-checking with daily use of acid-suppressant medication (60\% for cases and $0.06 \%$ for non-cases) demonstrated that the degree of contamination between cases and non-cases was likely to be minimal. The only other cohort study of diet and risk of GERD $^{(11)}$ compared symptoms 1 or more days per week with less frequent symptoms and found no association for any dietary exposures, possibly due to misclassification of the outcome. In our sensitivity analysis in which the case definition was relaxed to symptoms on $\geq 1 \mathrm{~d}$ /week, the observed associations were attenuated.

A limitation of the study is the overlap in symptoms between GERD and other gastrointestinal conditions ${ }^{(30)}$, which could explain some of the associations observed. For example, functional dyspepsia is characterised by both non-GERD-related (e.g. fullness and bloating) and GERDrelated symptoms ${ }^{(31)}$. Red meat has been associated with symptoms of fullness but not with other dyspeptic symptoms ${ }^{(32,33)}$ and was avoided by patients in a case-control study of functional dyspepsia ${ }^{(33)}$. It is plausible that these people have higher chicken and seafood consumption. Eosinophilic oesophagitis could also exhibit GERD-related symptoms, and dietary interventions such as the six-food elimination diet (milk, eggs, nuts, seafood, soy and wheat) have shown to be effective for disease remission ${ }^{(34)}$. In our study, no evidence for association was observed for total, red and processed meat, whereas fish and chicken were 
associated with increased risk of GERD. These associations were attenuated after excluding participants who reported following a special diet at baseline. Symptom overlaps between GERD and irritable bowel syndrome could partly explain the association observed for cruciferous vegetables. An estimated 5-25\% of people worldwide suffer from irritable bowel syndrome, which overlaps with GERD in 5-30\% of community-based individuals ${ }^{(30)}$. Some cruciferous vegetables contain high levels of Fermentable Oligosaccharides, Disaccharides, Monosaccharides and Polyols, which are known to trigger irritable bowel syndrome ${ }^{(35)}$.

Our findings are applicable to populations with similar Western-style diets to participants of the Melbourne Collaborative Cohort Study. Findings may be less applicable to populations where the Western-style diet is not as common, although diet is becoming more 'westernized' globally. We examined the associations in middle-age people, which might limit generalisability to other age groups.

\section{Sex-specific associations}

Women $(5.9 \%)$ were more likely to develop daily symptoms than men (3.9\%); however, nutrient intakes showed no associations for women. A proposed mechanism for GERD involves increased episodes of inappropriate transient relaxation of the lower oesophageal sphincter (LES) accompanied by reflux of gastric contents ${ }^{(36)}$. Oestrogen might contribute to reflux symptoms by lowering the LES tone and inhibiting gastric emptying ${ }^{(37-39)}$. Thus, while increased fat or decreased starch intake might have contributed to the pathogenesis of GERD in men, it might not contribute to the already elevated risk for women. Effect modification by sex might explain conflicting findings on the effect of fat on the LES. A trial with ten healthy men found LES pressure was lower after ingestion of a high-fat meal $^{(40)}$, whereas two other small trials with approximately equal numbers of men and women found no significant difference in LES pressure after fat ingestion ${ }^{(41,42)}$.

\section{Types of fat intake}

All types of fat intake were associated with risk of GERD for men, whereas in cross-sectional studies, only saturated fat intake was associated with reflux symptoms ${ }^{(24,25)}$. This suggests that while saturated fat in particular may trigger symptoms in established GERD, any type of fat could contribute to the development of GERD in men.

\section{Diet as trigger $v$. risk factor}

Our findings highlight that food commonly considered as triggers of GERD symptoms might not necessarily contribute to disease development. Citrus, tomato and coffee are considered triggers of symptoms ${ }^{(43,44)}$. In our study, tomato and coffee were not associated with the risk of developing GERD, while citrus had an inverse association. A trial that investigated heartburn symptoms following infusion of orange juice, tomato juice and coffee demonstrated that patients experienced symptoms even when the acidity of the solution was altered to neutral $(\mathrm{pH} 7)$, suggesting that the acidity of these items was not a cause of symptoms ${ }^{(45)}$.

Carbonated beverages were associated with increased risk of GERD and are a commonly cited trigger for GERD symptoms ${ }^{(46,47)}$. This could be explained by the carbon dioxide present in these beverages, which has been linked to increased belching and inappropriate LES relaxation ${ }^{(48)}$. It was unclear whether these beverages were caffeinated. However, given the lack of association observed for coffee, it is unlikely that caffeine would explain the association observed for carbonated beverages.

\section{Dietary patterns}

We found no evidence of association between the MDS or the AHEI-2010 and risk of GERD. A cross-sectional study in a south-eastern European population found an inverse association between Mediterranean diet and the presence of GERD ${ }^{(49)}$. The lack of association in our study could be due to the contrasting association between components of the dietary scores and GERD, which offset each other. For instance, fish was associated with increased risk of GERD, but is a 'beneficial' component in the MDS, whereas dairy was associated with reduced risk of GERD but is considered a 'harmful' component in the $\operatorname{MDS}^{(15)}$.

\section{Conclusion}

In conclusion, our prospective analysis suggests that some dietary components are possible risk factors for GERD, but diets conventionally considered healthy appear unlikely to reduce its risk. Food commonly considered as triggers of GERD symptoms might not necessarily contribute to the development of the disease. The potential differential associations for men and women warrant further investigation.

\section{Acknowledgements}

Acknowledgements: We thank David Whiteman for assistance with developing the questionnaire. Financial support: The Melbourne Collaborative Cohort Study (MCCS) cohort recruitment was funded by VicHealth and Cancer Council Victoria. The MCCS was further augmented by Australian National Health and Medical Research Council grants 209057, 396414 and 1074383 and by infrastructure provided by Cancer Council Victoria. Cancer cases and vital status of participants were ascertained through the Victorian Cancer Registry and the Australian Institute of Health and Welfare, including the National Death Index and the Australian Cancer Database. The study of gastro-oesophageal reflux was funded by NHMRC project grant (504708). S.W. is supported by an Australian Government Research 
Training Program Scholarship. Conflict of interest: The authors have no conflict of interest to declare. Authorship: S.E.W., A.M.H. and S.G.D. were involved in research question formulation, data analysis, results interpretation and article revision; S.C.D.-S. and B.J.K. were involved in results interpretation and article revision; H.M. was involved in data acquisition; R.J.S.T., A.B. and A.M.H. were involved in obtaining financial support and article revision; E.M.W. and R.L.M. revised the article; E.M. was involved in data analysis and article revision; G.G.G. was involved in obtaining financial support and article revision; D.R.E. was involved in research question formulation, financial support and data acquisition, data analysis, results interpretation and article revision. Ethics of buman subject participation: This study was conducted according to the guidelines laid down in the Declaration of Helsinki, and all procedures involving study participants were approved by the Human Research Ethics Committee at Cancer Council Victoria. Written informed consent was obtained from all participants.

\section{Supplementary material}

For supplementary material accompanying this paper visit https://doi.org/10.1017/S1368980021000197

\section{References}

1. Vakil N, van Zanten SV, Kahrilas P et al. (2006) The Montreal definition and classification of gastroesophageal reflux disease: a global evidence-based consensus. Am J Gastroenterol 101, 1900-1920.

2. Vakil N (2010) Disease definition, clinical manifestations, epidemiology and natural history of GERD. Best Pract Res Clin Gastroenterol 24, 759-764.

3. Revicki DA, Wood M, Maton PN et al. (1998) The impact of gastroesophageal reflux disease on health-related quality of life 1. Am J Med 104, 252-258.

4. Ronkainen J, Aro P, Storskrubb T et al. (2006) Gastrooesophageal reflux symptoms and health-related quality of life in the adult general population - the Kalixanda study. Aliment Pharmacol Ther 23, 1725-1733.

5. Coleman HG, Xie SH \& Lagergren J (2018) The epidemiology of esophageal adenocarcinoma. Gastroenterology 154, 390-405.

6. Eusebi LH, Ratnakumaran R, Yuan Y et al. (2017) Global prevalence of, and risk factors for, gastro-oesophageal reflux symptoms: a meta-analysis. Gut 67, 430-440.

7. Department of Health (2019) Pharmaceautical Benefits Scheme Information Management Section PBS Expenditure and Prescriptions [Internet]. Canberra: Department of Health; available at http://www.pbs.gov.au/info/statistics/ expenditure-prescriptions/pbs-expenditure-and-prescriptions (accessed December 2019).

8. Shaheen NJ, Hansen RA, Morgan DR et al. (2006) The burden of gastrointestinal and liver diseases, 2006. Am J Gastroenterol 101, 2128-2138.

9. Napier KJ, Scheerer M \& Misra S (2014) Esophageal cancer: a review of epidemiology, pathogenesis, staging workup and treatment modalities. World J Gastrointest Oncol 6, $112-120$
10. Schneider JL \& Corley DA (2017) The troublesome epidemiology of Barrett's esophagus and esophageal adenocarcinoma. Gastrointest Endosc Clin N Am 27, 353-364.

11. Zheng Z, Nordenstedt H, Pedersen NL et al. (2007) Lifestyle factors and risk for symptomatic gastroesophageal reflux in monozygotic twins. Gastroenterology 132, 87-95.

12. Milne RL, Fletcher AS, MacInnis RJ et al. (2017) Cohort Profile: the Melbourne Collaborative Cohort Study (Health 2020). Int J Epidemiol 46, 1757-1757i.

13. Australian Bureau of Statistics (2018) Technical Paper: Census of Population and Housing: Socio-Economic Indexes for Areas (SEIFA), 2016. ABS Cat. No. 2033.0.55.001. Canberra: ABS.

14. Hunt R, Armstrong D, Katelaris P et al. (2017) World gastroenterology organisation global guidelines: GERD global perspective on gastroesophageal reflux disease. J Clin Gastroenterol 51, 467-478.

15. Trichopoulou A, Costacou T, Bamia C et al. (2003) Adherence to a Mediterranean diet and survival in a Greek population. N Engl J Med 348, 2599-2608.

16. Hodge AM, English D, Itsiopoulos C et al. (2011) Does a Mediterranean diet reduce the mortality risk associated with diabetes: evidence from the Melbourne Collaborative Cohort Study. Nutr Metabol Cardiovasc Dis 21, 733-739.

17. Chiuve SE, Fung TT, Rimm EB et al. (2012) Alternative dietary indices both strongly predict risk of chronic disease. J Nutr 142, 1009-1018.

18. Rothman KJ, Greenland S \& Lash TL (2008) Modern Epidemiology, 3rd ed. Philadelphia: Lippincott Williams \& Wilkins.

19. Seaman SR \& White IR (2013) Review of inverse probability weighting for dealing with missing data. Stat Methods Med Res 22, 278-295.

20. Tibshirani R (2011) Regression shrinkage and selection via the lasso: a retrospective. J Roy Stat Soc Ser B (Stat Method) 73, 273-282.

21. R Core Team (2014) R: A Language and Environment for Statistical Computing. Vienna, Austria: R Foundation for Statistical Computing.

22. StataCorp (2015) Stata Statistical Software: Release 14. College Station, TX: StataCorp LP.

23. Willett W (2013) Nutritional Epidemiology. New York: Oxford University Press.

24. El-Serag HB, Satia JA \& Rabeneck L (2005) Dietary intake and the risk of gastro-oesophageal reflux disease: a cross sectional study in volunteers. Gut 54, 11-17.

25. Shapiro M, Green C, Bautista JM et al. (2007) Assessment of dietary nutrients that influence perception of intra-oesophageal acid reflux events in patients with gastro-oesophageal reflux disease. Aliment Pharmacol Ther 25, 93-101.

26. Nilsson M, Johnsen R, Ye W et al. (2003) Obesity and estrogen as risk factors for gastroesophageal reflux symptoms. JAMA 290, 66-72.

27. Nilsson M, Lundegårdh G, Carling L et al. (2002) Body mass and reflux oesophagitis: an oestrogen-dependent association? Scand J Gastroenterol 37, 626-630.

28. Pace F \& Porro GB (2006) Clinical spectrum, natural history and epidemiology of GERD. In Gastroesophageal Reflux Disease: Principles of Disease, Diagnosis, and Treatment, pp. 1-11 [FA Granderath, T Kamolz and R Pointner, editors]. Wien; New York: Springer.

29. Crowell MD, Zayat EN, Lacy BE et al. (2001) The effects of an inhaled $\beta 2$-adrenergic agonist on lower esophageal function. Chest 120, 1184-1189.

30. de Bortoli N, Tolone S, Frazzoni M et al. (2018) Gastroesophageal reflux disease, functional dyspepsia and irritable bowel syndrome: common overlapping gastrointestinal disorders. Ann Gastroenterol 31, 639-648. 
31. Feinle-Bisset C \& Azpiroz F (2013) Dietary and lifestyle factors in functional dyspepsia. Nature Rev Gastroenterol Hepatol 10, 150.

32. Filipović BF, Randjelovic T, Kovacevic N et al. (2011) Laboratory parameters and nutritional status in patients with functional dyspepsia. Eur J Intern Med 22, 300-304.

33. Carvalho RVB, Lorena SLS, de Souza Almeida JR et al. (2009) Food intolerance, diet composition, and eating patterns in functional dyspepsia patients. Digest Dis Sci 55, 60.

34. Arias Á, González-Cervera J, Tenias JM et al. (2014) Efficacy of dietary interventions for inducing histologic remission in patients with eosinophilic esophagitis: a systematic review and meta-analysis. Gastroenterology 146, 1639-1648.

35. Muir JG, Rose R, Rosella O et al. (2009) Measurement of short-chain carbohydrates in common Australian vegetables and fruits by high-performance liquid chromatography (HPLC). J Agric Food Chem 57, 554-565.

36. Mikami DJ \& Murayama KM (2015) Physiology and pathogenesis of gastroesophageal reflux disease. Surg Clin North Am 95, 515-525.

37. Fisher RS, Roberts GS, Grabowski CJ et al. (1978) Inhibition of lower esophageal sphincter circular muscle by female sex hormones. Am J Physiol Endocrinol Metabol 234, E243.

38. Van Thiel DH, Gavaler JS \& Stremple J (1976) Lower esophageal sphincter pressure in women using sequential oral contraceptives. Gastroenterology 71, 232-235.

39. Hutson WR, Roehrkasse RL \& Wald A (1989) Influence of gender and menopause on gastric emptying and motility. Gastroenterology 96, 11-17.

40. Nebel OT \& Castell DO (1973) Inhibition of the lower oesophageal sphincter by fat-a mechanism for fatty food intolerance. Gut 14, 270-274.
41. Penagini R, Mangano M \& Bianchi P (1998) Effect of increasing the fat content but not the energy load of a meal on gastro-oesophageal reflux and lower oesophageal sphincter motor function. Gut 42, 330-333.

42. Pehl C, Waizenhoefer A, Wendl B et al. (1999) Effect of low and high fat meals on lower esophageal sphincter motility and gastroesophageal reflux in healthy subjects. $\mathrm{Am} J$ Gastroenterol 94, 1192.

43. Sethi S \& Richter JE (2017) Diet and gastroesophageal reflux disease: role in pathogenesis and management. Curr Opin Gastroenterol 33, 107-111.

44. Feldman M \& Barnett C (1995) Relationships between the acidity and osmolality of popular beverages and reported postprandial heartburn. Gastroenterology 108, 125-131.

45. Price SF, Smithson KW \& Castell DO (1978) Food sensitivity in reflux esophagitis. Gastroenterology 75, 240-243.

46. Song JH, Chung SJ, Lee JH et al. (2011) Relationship between gastroesophageal reflux symptoms and dietary factors in Korea. J Neurogastroenterol Motil 17, 54-60.

47. Fass R, Quan SF, O'Connor GT et al. (2005) Predictors of heartburn during sleep in a large prospective cohort study. Chest 127, 1658-1666.

48. Hamoui N, Lord RV, Hagen JA et al. (2006) Response of the lower esophageal sphincter to gastric distention by carbonated beverages. J Gastrointest Surg 10, 870-877.

49. Mone I, Kraja B, Bregu A et al. (2016) Adherence to a predominantly Mediterranean diet decreases the risk of gastroesophageal reflux disease: a cross-sectional study in a South Eastern European population. Dis Esophagus 29, 794-800. 